\title{
Towards a Multimodal Adaptive Lighting System for Visually Impaired Children
}

\author{
Euan Freeman, Graham Wilson and Stephen Brewster \\ Glasgow Interactive Systems Group \\ University of Glasgow \\ Glasgow, Scotland \\ \{first.last\}@glasgow.ac.uk
}

\begin{abstract}
Visually impaired children often have difficulty with everyday activities like locating items, e.g. favourite toys, and moving safely around the home. It is important to assist them during activities like these because it can promote independence from adults and helps to develop skills. Our demonstration shows our work towards a multimodal sensing and output system that adapts the lighting conditions at home to help visually impaired children with such tasks.
\end{abstract}

\section{CCS Concepts}

-Human-centered computing $\rightarrow$ Accessibility technologies;

\section{Keywords}

Visual impaired; children; adaptive lighting; beacons.

\section{INTRODUCTION}

Activities like finding clothes and toys can be challenging for visually impaired children, especially younger children who are just beginning to learn about their surroundings and are becoming more active. Everyday activities can be difficult because visual impairment makes it challenging for them to identify items against cluttered and complex backgrounds. Lighting levels also affect their ability to see clearly; e.g., it is more difficult to see items in dark areas of rooms or when there are low levels of natural light [4]. Their ability to identify objects is also impaired by poor perception of shape and form; however, other visual properties like light [3], colour and motion [1] can be perceived more easily.

We have been investigating ways of automatically adapting the lights within the home so that more easily perceived visual stimulus - i.e., bright lights, colour and motion-can be used to help visually impaired children with everyday activities, like locating things or finding their way at home. Changing the lighting may also stimulate vision and encourage children to use their residual sight more. This is important because it helps develop their visual skills 2 .

Our research builds on existing strategies for adapting the home environment (e.g., increasing brightness of lights and simplifying decor) to help visually impaired children. However, we propose adapting the environment automatically, using a multimodal approach to activity and location sensing within the home. In this paper we describe how our proposed system would use data from beacons and wearables to determine where a child is, what they are doing, and what their lighting needs are. We begin by presenting our prototype system for sensing activity and adapting the lights at home. We then describe two scenarios which show how adaptive lighting may benefit visual impaired children, ending with a description of our demonstration.

\section{ADAPTIVE LIGHTING SYSTEM}

Our system is based on hardware developed by the ABBI (Audio Bracelet for Blind Interaction) project ${ }^{7}$, which is developing a smart bracelet for visually impaired children. The main purpose of the ABBI bracelet is to support sensorymotor rehabilitation, by producing sounds in response to movement; the project has developed successful rehabilitation protocols and activities based on this approach.

ABBI bracelets (Figure 1, Left) have rich sensing capabilities that mean they can also be used for other interactions, like the ones we consider in this paper. It has a microphone, a 9-axis inertial motion sensor, Bluetooth low energy, and a loudspeaker for on-board audio synthesis and playback. Bluetooth is used for communication with other devices and to allow the device to act as a beacon. Future versions of the firmware could extend these capabilities, e.g. to scan for other beacons as well. The features of the ABBI hardware identified here - current and future - allow the devices to support the functionality identified in the next section.

Our adaptive lighting system uses ABBI bracelets in two ways: (1) they can be worn by a child or placed on toys to provide motion sensor data, which can be used for basic activity detection (e.g., detecting movement, walking, inactivity, etc.); and (2) they can be placed in rooms and used as beacons. This allows a multimodal approach to estimating a child's position within the home as well as their activity level, which informs our lighting system and supports decision making about how the lighting should be configured.

ABBI hardware provides input and auditory output. Our other output modality - the lighting within the home - is provided by smart light bulbs, which can be controlled over

\footnotetext{
$\sqrt[1]{\text { https://www.abbiproject.eu/ }}$
} 

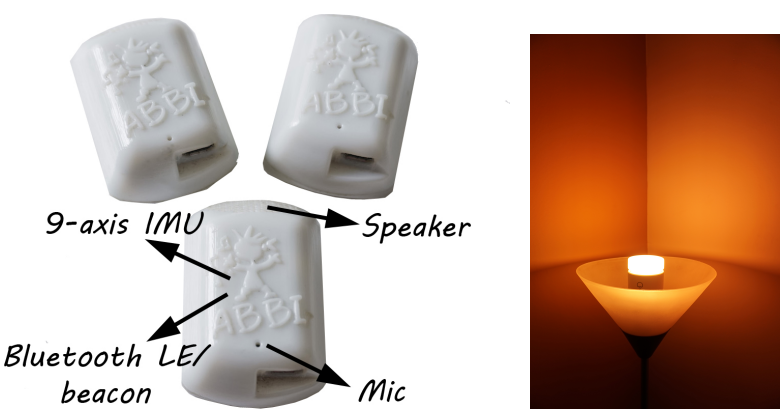

Figure 1: (Left): 3D-printed ABBI devices. The straps can be removed to allow the devices to be placed in rooms as beacons or can be added to toys or other items to give them sensing capabilities; (Right): A LIFX lightbulb in a standing lamp.

wifi. We use LIFX lightbulbs ${ }^{2}$ (see Figure 1 Right) that fit in standard light fittings and support a wide range of hues and intensities. We use a smartphone to scan for beacons, process motion data from ABBI devices, and control the lights; this allows us to rapidly prototype new interactions without reprogramming the ABBI micro-controller.

\section{ADAPTIVE LIGHTING SCENARIOS}

\subsection{Safe Movement \& Supporting Play}

Jane needs bright light to help her move safely around the house. As she enters her bedroom, the lights turn on at full brightness so she can see furniture and obstacles. A coloured lamp turns on against the far wall, emitting a green light to illuminate the area where her toys are. She walks over to her toys and sits down. When she picks up a light-up toy with an ABBI inside it, the lights are dimmed, to make it easier for her to see the toy. As she stands up to leave, the brightness increases to help her find a clear path.

In this scenario, the lighting system increased the brightness of the lights to help Jane see obstacles and furniture in her bedroom. It also used colour and motion to attract her attention towards her toys, supporting an association between colours and tasks 1 which is beneficial when items are difficult to perceive. The lighting system also dimmed the lights so that visually-stimulating toys were easier to see. Children with visual impairments are encouraged to play with toys which light up, as this can stimulate their sight, so dimming the lights can enhance this type of play.

\subsection{Attracting Attention \& Stimulating Vision}

Tom's mother wants him to try playing with new toys, to encourage him to explore his surroundings more. She places an ABBI inside a new toy, so that a lamp in the room creates colourful patterns when he plays with the toy. When Tom walks into the playroom, the ABBI in the toy uses sound to attract his attention. As he lifts and moves the toy, the light from the lamp changes, creating exciting colourful patterns on the wall. The ABBI also makes sounds as Tom plays.

In this scenario, the lighting system used sound to catch Tom's attention and make him aware of the toy his mother placed in the room. As he picked it up and moved it, light

\footnotetext{
$\sqrt[2]{\text { http://www.lifx.com/ }}$
}

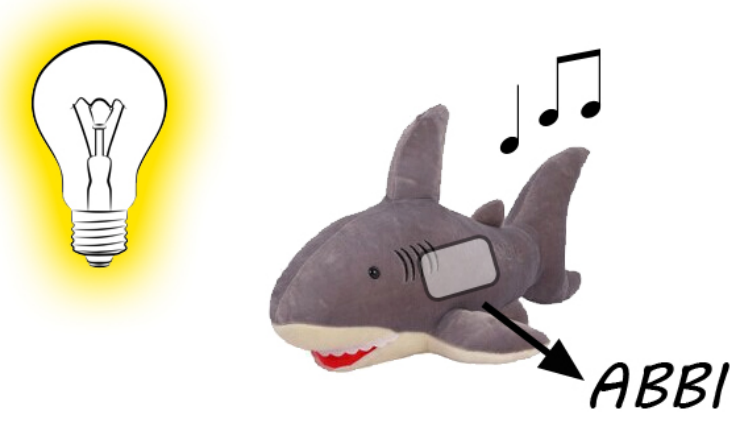

Figure 2: Demo hardware - a soft toy with an ABBI inside that acts as a beacon, senses motion and synthesises sound; and a 'smart' light source.

from the lamp changed, creating exciting visual stimulus. Stimulating vision is important as it can improve sight, as mentioned before. Creating visual and auditory stimulus in response to the toy's movement can also be beneficial, as it can 'reward' curiosity and helps teach about 'cause and effect' relationships, which is important in early development of the brain, especially for visually impaired children.

\section{DEMONSTRATION DESCRIPTION}

We will demonstrate functionality described in the second scenari ${ }^{3}$ and shown in Figure 2 as a user approaches a stuffed toy (with an ABBI inside of it), the toy will make noise to attract attention; then when the toy is lifted and moved, a small lamp (similar to Figure 1, Right) will change colour, pulse, and glow, creating exciting visuals. The ABBI will also make sound in response to movement.

This demo will show the versatility of the ABBI bracelets: they can be used for multimodal input (beacon proximity \& motion) and output (sound \& lights). Our demo also shows a novel combination of Bluetooth beacons and interactive lighting, new technologies that have not been used together in this type of accessible interaction before.

\section{ACKNOWLEDGMENTS}

This work is funded by the EU ABBI project (\#611452).

\section{REFERENCES}

[1] S. A. Cohen-Maitre and P. Haerich. Visual Attention to Movement and Color in Children with Cortical Visual Impairment. Journal of Visual Impairment $\mathcal{E}$ Blindness, 99(7):1-20, 2005.

[2] E. Fazzi, S. G. Signorini, S. M. Bova, P. Ondei, and P. E. Bianchi. Early intervention in visually impaired children. International Congress Series, 1282:117-121, 2005.

[3] N. M. Flanagan, A. J. Jackson, and A. E. Hill. Visual impairment in childhood: Insights from a community-based survey. Child: Care, Health and Development, 29(6):493-499, 2003.

[4] L. Hyvärinen. Development of Vision Impaired Children without Other Impairments, 2011.

\footnotetext{
${ }^{3}$ See http://www.youtube.com/watch?v=f4LkuCmfehg for
} a video of some functionality from the first scenarı. 